\title{
Técnicas electroquímicas cuali y cuantitativas para medir los efectos de las adiciones sobre la corrosión de las armaduras*

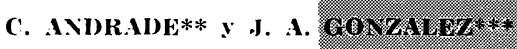

\section{INTRODUCCION}

La utilización creciente de adiciones activas, como constituyente de los cementos en sustitución de una parte del clínker de cemento portland (los cementos PA), ha desencadenado en nuestro país una problemática diversa que todavía suscita abundante polémica entre fabricantes y usuarios.

La posible incidencia específica de los cementos con adiciones activas en la corrosión de armaduras es un aspecto de esa problemática que ha llevado a especulaciones e hipótesis y, en general, a una cierta desconfianza de los usuarios, en la tradicional capacidad protectora del cemento hacia las armaduras y las tuberías metálicas en general.

En el presente trabajo se resumen, primeramente, una serie de consideraciones teóricas sobre la incidencia que las adiciones activas pueden tener en la corrosión de las armaduras; después, se llama la atención sobre la ausencia de normativas o recomendaciones de trabajo, sustentadas en ensayos suficientes, que permitan delimitar las causas y condiciones que influyen sobre la durabilidad de las armaduras; finalmente se expone una sistemática de ensayos que se ha llevado a cabo para estudiar esta incidencia mediante técnicas electroquím:cas y las diferencias de comportamiento encontradas entre los distintos cementos.

\section{Consideraciones teóricas}

La hidratación de los silicatos cálcicos del clínker de cemento portland libera gran cantidad de $\mathrm{Ca}(\mathrm{OH})_{2}$ que se disuelve en una pequeña proporción (alrededor de $1,2 \mathrm{gr} / \mathrm{l}$ a $20-25^{\circ} \mathrm{C}$ ) dando lugar a un $\mathrm{pH} \simeq 12,6$ en la fase líquida contenida en los poros del hormigón. La mayor parte de este $\mathrm{Ca}(\mathrm{OH})_{2}$ permanece precipitado constituyendo la llama-

" Una versión de este trabajo ha sido presentado al Symposium sobre "Laitiers et ciments aux ajouts". Celebrado en Mons (Bélgica) en septiembre de 1981.

*: C. Andrade. Dr. en Química Industrial. Colaborador Cientifico del Instituto Eduardo Torroja, C.S.I.C. Madrid.

*** J. A. González. Dr. en Quimica Industrial. Investigador Científico del CENIM. C.S.I.C. Madrid. 
da "reserva alcalina". Los álcalis $\left(\mathrm{Na}^{*}\right.$ y $\left.\mathrm{K}^{*}\right)$ presentes en el clínker contribuyen también al $\mathrm{pH}$ de esta fase líquida, elevándolo en ocasiones por encima de 13 (1) (2).

Este elevado $\mathrm{pH}$ del agua contenida en los poros del horm:gón y el oxígeno disuelto en ella, garantizan la pasividad de las armaduras en tanto no se modifiquen las condiciones de servicio.

Los diversos tipos de adiciones activas añadidas al clínker reaccionan por un mecanismo u otro - según sea el tipo de adición-, con la mencionada reserva alcalina del cemento hidratado para formar silicatos, aluminatos y ferritos cálcicos, lo que si, por un lado mejora la resistencia físicoquímica (e incluso la mecánica) de la pasta frente a determinados ambientes agresivos, por otro, supone una cierta disminución de la tradicional capacidad protectora del cemento hacia las armaduras, circunstancia que puede obligar a adoptar, ante un determinado medio agresivo, una solución de compromiso entre un cemento que asegure la mayor estabilidad físico-química al conglomerante y el que proporcione la mayor protección a las armaduras.

Si las adiciones utilizadas son de las llamadas tradicionalmente "inertes", el efecto desfavorable hacia las armaduras vendría propiciado fundamentalmente por el menor contenido porcentual de clínker de portland en el cemento, con la consiguiente disminución de la reserva alcalina.

Esta disminución de la capacidad protectora no significa un ataque a las armaduras (a excepción de los $\mathrm{S}^{2-}$ que contienen las escorias; las adiciones activas no contienen agentes desencadenantes de corrosión), como lo prueba la multiud de estructuras realizadas con cementos de adición con largos años de vida en servicio y el hecho de que el $\mathrm{pH}$ de las pastas de estos cementos se mantiene en niveles de basicidad adecuados (3), sino que supone un aumento del riesgo de corrosión, es decir, que el umbral o energía necesaria para que se desencadene un determinado ataque es menor en los cementos con adición, que en los portland puros.

\section{Necesidad de una clasificación o normativa}

La constatación de que no todos los cementos confieren un mismo nivel de protección al acero hace necesaria la definición de unos índices que permitan clasificar los cementos y delimitar su campo de aplicación en función del grado de agresividad del ambiente.

Tal es la situación del hormigón en masa para el que existen en la actualidad numerosos métodos que permiten estudiar su durabilidad fis.coquímica y, a pesar de que estos métodos de ensayo no siempre aportan indicaciones coincidentes, se puede llegar con cierta aproximación a unas recomendaciones de uso de los cementos, en función de su resistencia al ataque.

Esta situación no tiene paralelo similar en el caso de la durabilidad de las armaduras (tal vez porque hasta hace muy pocos años no se ha contado con una técnica de medida de la velocidad de corrosión que, como la determinación de la Resistencia de Polarización, $R_{p}$, permite un conocimiento más profundo de los mecanismos de este tipo de alteración). Sólo se cuenta en la actualidad con recomendaciones amplias (4) (5) y con diversas recopilac:ones de estudios y ensayos (6) pero efectuados en condiciones de trabajo muy dispersas.

Este hecho indica la necesidad de delimitar las causas y condiciones que influyen sobre el riesgo de corrosión de las armaduras, y no sólo en función del tipo de cemento utilizado, sino contemplando interrelacionadas todas las circunstancias que pueden desenca- 
denar este tipo de ataque. En este sentido se considera valiosa la propuesta efectuada en (7) donde se define el riesgo de corrosión de las armaduras (sin tesar) como el resultado de la conjugación simultánea durante la vida en servicio de la estructura de:

A) la susceptibilidad a la corrosión del sistema armadura-hormigón, que vendría a su vez determinada por:

- tipo y acabado superficial del acero,

- tipo de cemento,

- dosificación del hormigón,

- permeabilidad del hormigón,

- fisuración.

B) la agresividad del medio, para lo que habría que considerar principalmente:

- presencia de contaminantes: $\mathrm{Cl}^{-}, \mathrm{CO}_{2}$ y derivados del azufre $\left(\mathrm{SO}_{2}, \mathrm{~S}^{=}\right.$y $\left.\mathrm{SO}_{4}{ }^{=}\right)$,

- grado de humidificación del hormigón.

Sería necesario cuantificar la incidencia de cada una de estas variables por separado [labor efectuada ya en algunos casos (8) (9)] y luego estudiar las interrelaciones mutuas para, finalmente, poder llegar a definir un índice o nivel de protección o resistencia a la corrosión de un determinado sistema: acero/cemento/hormigón/medio.

\section{ENSAYOS REALIZADOS Y TECNICAS EXPERIMENTALES}

Se presentan aquí algunos de los ensayos realizados para estudiar la influencia que ejerce el tipo de cemento y, de forma secundaria, el acabado superficial del acero sobre la corrosión de las armaduras.

Con la finalidad de poder atribuir un nivel o índice de capacidad protectora a cada cemento, se elaboró una sistemática de ensayos que se ha resumido en el cuadro I, y que si bien hasta el momento ha parecido la más apropiada, no se descarta la posibilidad de ampliarla o perfeccionarla en el futuro.

C UADRO I

\begin{tabular}{|c|l|l|}
\hline CONDICIONES & MEDIDAS QUE SE EFECTUAN & DURACION DE LOS ENSAYOS \\
\hline \multirow{2}{*}{$\begin{array}{c}\text { Mortero sin aditivos conservado } \\
\text { parcialmente sumergiclo. }\end{array}$} & $\begin{array}{c}\text { Determinación de la } I_{\text {corr }} \text { a partir } \\
\text { de } R_{\mathrm{p}} .\end{array}$ & 28 días. \\
\cline { 2 - 4 } $\begin{array}{c}\text { Evaluación de } E_{\mathrm{r}} \text { mediante cur- } \\
\text { vas de polarización. }\end{array}$ & $2-7 \mathrm{y} 28$ días. \\
\hline $\begin{array}{c}\text { Mortero con } 2 \% \mathrm{Cl}_{2} \mathrm{Ca} \text { conser- } \\
\text { vado parcialmente sumergido. }\end{array}$ & $\begin{array}{c}\text { Determinación de la } I_{\text {corr }} \text { a partir } \\
\text { de } R_{\mathrm{p}} .\end{array}$ & 28 días. \\
\cline { 2 - 4 } & $\begin{array}{c}\text { Evaluación de } E_{\mathrm{r}} \text { mediante cur- } \\
\text { vas de polarización. }\end{array}$ & $2-7 \mathrm{y} 28$ días. \\
\hline
\end{tabular}

En síntesis se trata de estudiar mediante técnicas electroquímicas la susceptibilidad a la corrosión y la cuantía de ésta, cuando se produce. 
Para ello se estudia con cada cemento:

a) el comportamiento del acero en ausencia de ataque, es decir, en un mortero sin aditivos;

b) el comportamiento del mismo acero, cuando se desarrolla un proceso de corrosión por uno de los dos mecanismos básicos que pueden darse en el hormigón armado: ataque por iones despasivantes (cloruros).

Las características de las probetas, su elaboración y curado se han expuesto con detalle en anteriores trabajos (10) (11).

\section{Técnicas de medida}

La velocidad de corrosión se estimó, durante 28 días, en un mortero conservado parcialmente sumergido en agua destilada mediante la medida de la Resistencia de Polarización, $R_{\mathrm{p}}$, de las armaduras (8) (11), técnica que permite el cálculo de la intensidad de corrosión, $I_{\text {corr }}$, instantánea, y con la posterior integración de la curva $I_{\text {corr-tiempo se }}$ puede calcular la pérdida de peso sufrida por el acero.

Los autores utilizan esta técnica de medida desde 1973 (12), logrando a través de sus indicaciones conocer con más profundidad el mecanismo de corrosión de las armaduras embebidas directamente en mortero u hormigón.

La probabilidad o susceptibilidad al ataque se ha evaluado con el trazado de curvas de polarización (potencio $\mathrm{y} / \mathrm{o}$ intensiostáticas), que revelan si se produce un potencial de ruptura, $E_{\mathrm{r}}$, y su situación, pudiendo estimarse que la susceptibilidad al ataque disminuye al aumentar la diferencia entre el $E_{\text {corr }}$ y el $E_{\mathrm{r}}$. Debido al carácter destructivo de estos métodos, es necesario utilizar una probeta nueva para cada ensayo.

A causa de la elevada resistividad del mortero son diversas las posibilidades de error que se presentan en el trazado de estas curvas y que es necesario tener presentes a la hora de extraer las conclusiones (8) (9) (10) (11). Para soslayar o minimizar este problema es necesario utilizar potenciostatos con eliminación instrumental de la caída óhmica y conservar las probetas muy húmedas (parcialmente sumergidas). Sólo se deben efectuar comparaciones con materiales y condiciones de trabajo y ensayo, absolutamente idénticas.

Las curvas de polarización han sido frecuentemente utilizadas para el estudio de la incidencia de aditivos con cloruros en la corrosión de armaduras (1) (3) (13) (14) (15). Sin embargo, se han utilizado menos frecuentemente para estudiar la influencia de las adiciones activas y, sobre todo, es original su valoración paralela con resultados de cinética de corrosión en condiciones no forzadas de conservación del mortero (10).

\section{RESULTADOS}

En la figura 1 se muestra el estudio realizado con un acero corrugado de dureza natural embebido en mortero fabricado con los siguientes cinco tipos de cemento:

n. 1 - Portland puro.

n. ${ }^{\circ} 2$ - Portland con $25 \%$ de puzolana especial.

n. 3 - Portland con $35 \%$ de puzolana especial.

n. 4 - Portland con $45 \%$ de puzolana especial.

n. 5 - Puzolánico (40\% puzolana). 

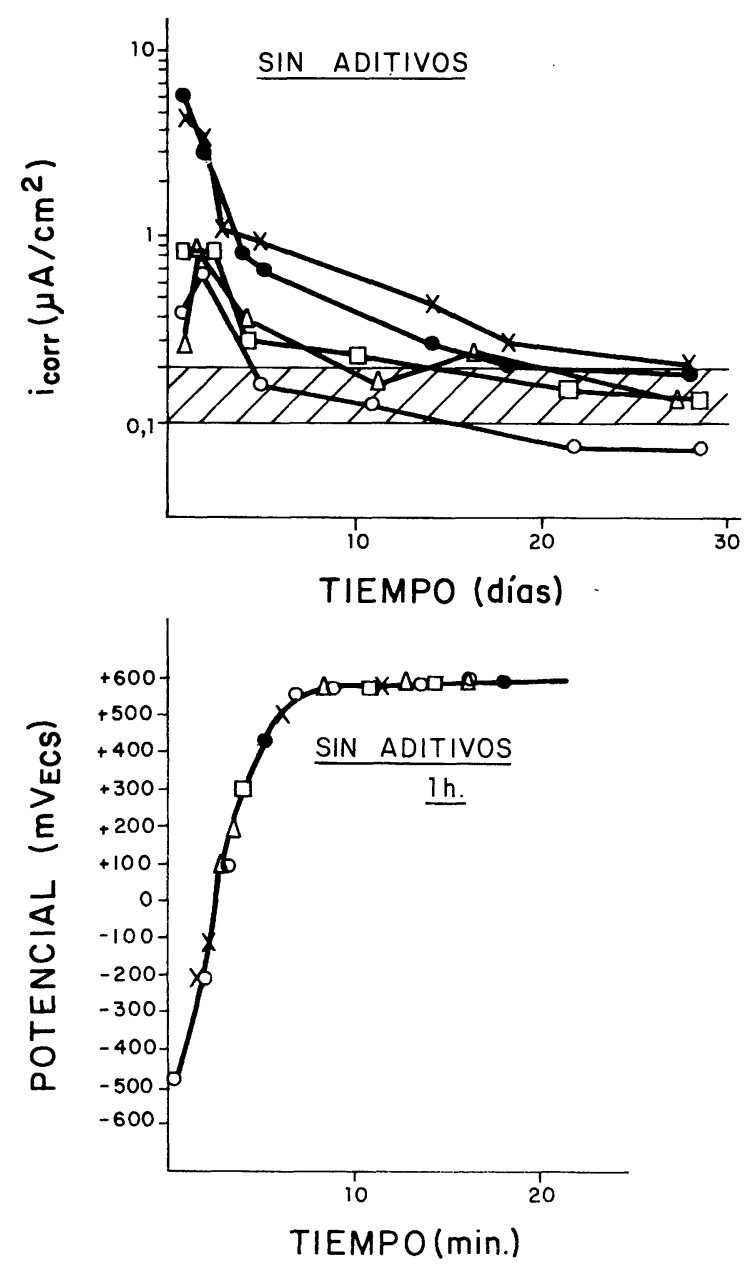
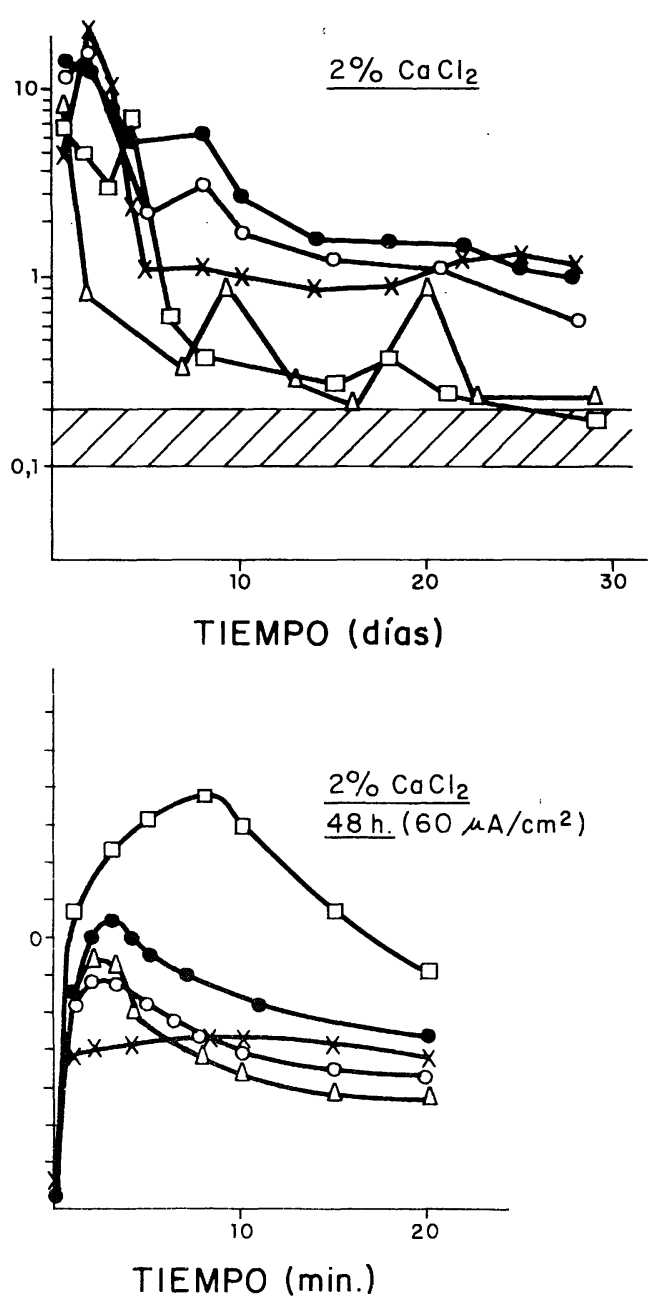

CEMENTOS

$1-\bullet-P$
$2-X-P$ con $25 \%$ puz. especial
$3-\Delta-P$ con $35 \%$ puz especial
$4-\square-P$ con $45 \%$ puz. especial
$5-0-P U z$

Fig. 1

En la parte superior de la figura se muestra la variación de la $I_{\text {corr }}$ con el tiempo de los cinco cementos, tanio sin aditivos como con $2 \% \mathrm{de}^{-\mathrm{CaCl}_{2}}$. Y en la parte inferior se han representado las curvas de polarización intensiostáticas $\left(60 \mu \mathrm{A} / \mathrm{cm}^{2}\right)$, de los mismos cinco cementos, a 1 hora (sin aditivos) y 48 horas $\left(2 \%\right.$ de $\left.\mathrm{CaCl}_{2}\right)$ de fabricación.

Mediante la consideración de la evolución de la $I_{\text {corr }}$ con el tiempo puede comprobarse que, en el mortero sin aditivos (parte izquierda de la figura), todos los cementos pasivan oorrectamente al acero, ya que en la curva de polarización (parte inferior izquierda) no aparece ningún $E_{\mathrm{r}}$ (si el cemento contiene $\mathrm{S}^{=}$se detecta en este ensayo por la aparición de un $E_{\mathrm{r}}$ ) y que los valores de $I_{\text {corr }}$ a los 28 días están todos dentro de la pasividad (la zona límite entre corrosión y pasividad se ha rayado en la figura).

En cambio, cuando el mortero contiene un $2 \% \mathrm{de} \mathrm{Cl}_{2} \mathrm{Ca}$ (parte derecha de la figura) los valores de $I_{c o r r}$ indican que el cemento n. $^{\circ} 4$ (portland con $45 \%$ de puzolana no tradicional) es el que menos se corroe, presentando a los 28 días unos valores próximos a la 
pasividad, mientras que los cementos 1 y 2 se corroen casi 10 veces más que el anterior. En cuanto a la susceptibilidad al ataque puede comprobarse que el cemento que induce $E_{r}$ más distanciado del $E_{\text {corr }}$ es también el n. ${ }^{\circ}$, siendo el menos protector frente al ataque de los $\mathrm{Cl}^{-}$el n. ${ }^{\circ}$.

Fn la figura 2 se ha representado un estudio similar al anterior efectuado con el mismo tipo de acero y con los cementos:

n. 1 - Puzolánico.

n. 2 - Portland con bajo contenido en $\mathrm{C}_{3} \mathrm{~A}$.

n. 3 - Portland normal.
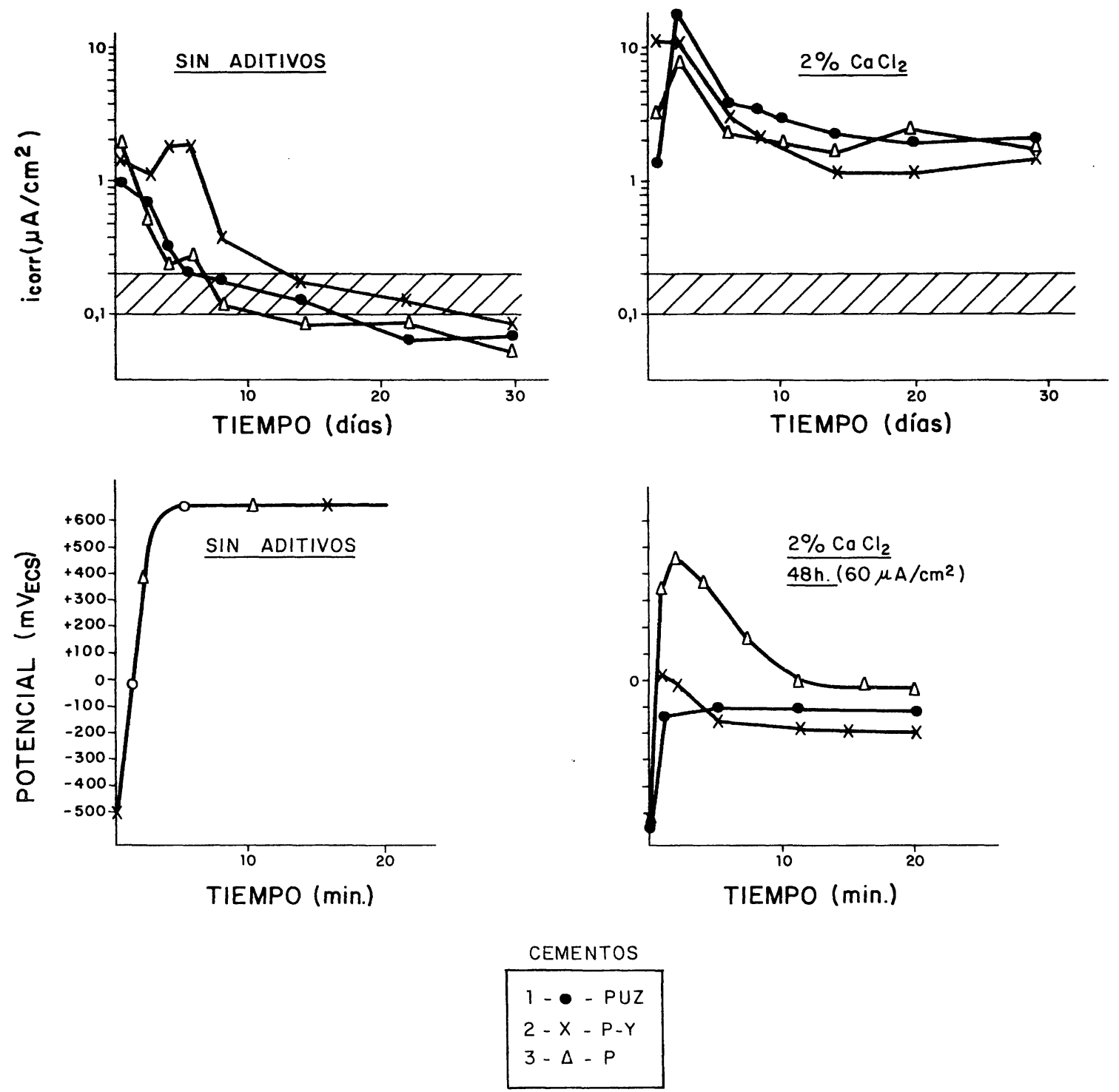

Fig. 2

Igualmente puede comprobarse que en el mortero sin aditivos (parte izquierda de la figura) no aparece ningún $E_{\mathrm{r}}$ en las curvas galvanostáticas, y que los valores de $I_{\text {corr }}$ que se alcanzan a los 28 días indican que todos los cementos pasivan correctamente al acero.

Cuando el cemento contiene un $2 \% \mathrm{CaCl}_{2}$ (parte derecha de la figura) puede comprobarse en los valores de da $I_{\text {corr }}$ que los tres cementos presentan una capacidad protectora similar, con valores bastante elevados si se comparan con los cementos de la figura 
anterior. De las curvas galvanostáticas se deduce que el cemento $\mathrm{n}^{\circ} 3$ (portland puro) . es el más protector de los tres ensayados, siendo el menos protector el puzolánico.

En la figura 3 se muestra otro estudio efectuado con el mismo tipo de acero y los siguientes cinco cementos:

1. Portland normal.

2. Portland con bajo contenido en $\mathrm{C}_{\mathbf{3}} \mathrm{A}$.

3. Cemento con $35 \%$ de escorias especiales.

4. Puzolánico.

5. Cemento con $35 \%$ de escorias.
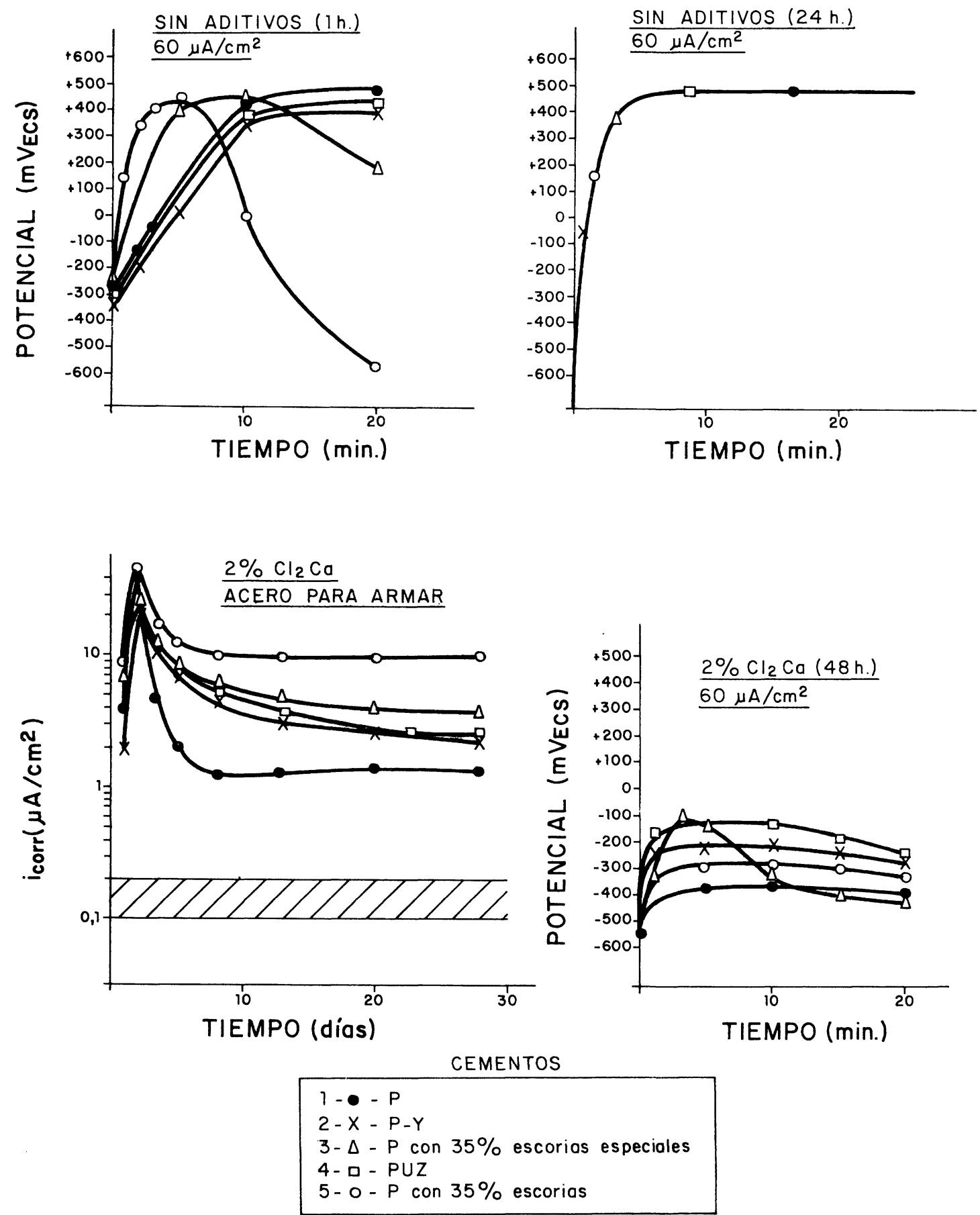

Fig. 3 
Se puede comprobar que el mortero sin aditivos los morteros fabricados a base de cemento con escorias (n. 3 y n. ${ }^{\circ}$ ) presentan un $E_{r}$ en las curvas galvanostáticas determinadas a los pocos minutos del amasado. Este potencial de ruptura es provocado probablemente por el contenido en $\mathrm{S}^{=}$de las escorias. En las curvas efectuadas a las 24 horas del amasado ya no aparece ningún potencial de ruptura, lo que indica que la acción despasivante de los $\mathrm{S}^{=}$ha sido bloqueada, o bien que estos iones se han combinado o se han oxidado para formar otros compuestos no agresivos.

En el mortero con $2 \% \mathrm{Cl}_{2} \mathrm{Ca}$ puede apreciarse que el cemento $n .{ }^{\circ} 5$ es el que presenta una cinética de corrosión más elevada, del orden de 10 veces la del cemento n.' 1 (portland puro) y 50 veces por encima del valor considerado como de pasividad.

Las curvas de polarización muestran también que el cemento n. ${ }^{\circ} 5$ presenta un $E_{\mathrm{r}}$ bastante próximo al $E_{\text {corr }}$. Asimismo, de estas curvas de la figura 3 se deduce que el cemento n. 1 es también muy susceptible al ataque por $\mathrm{Cl}^{-}$, a pesar de que su velocidad de corrosión sea la menos intensa.

Finalmente en la figura 4 se han representado los resultados obtenidos con los mismos cinco cementos de la figura 3 , sólo para el caso del mortero con $2 \% \mathrm{Cl}_{2} \mathrm{Ca}$, pero utilizando como armadura acero liso para pretensado en lugar de acero corrugado para armar.
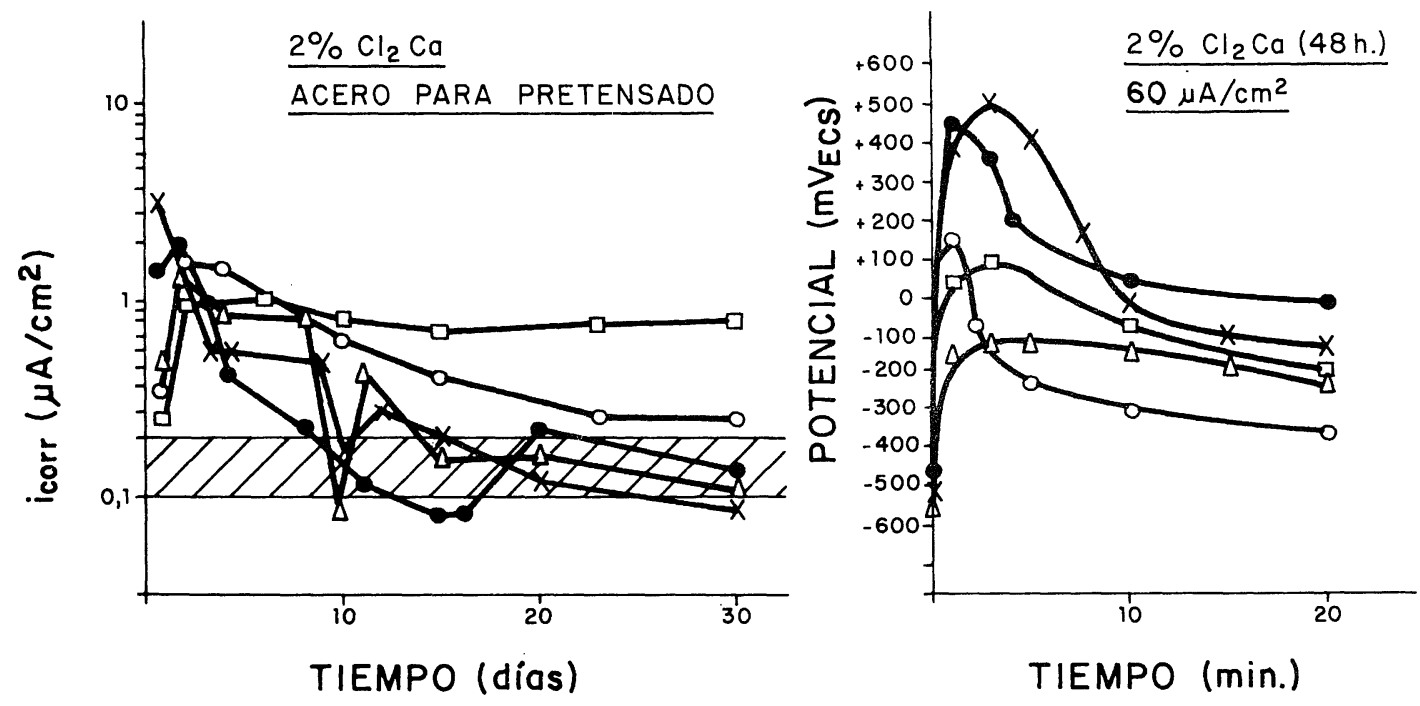

Fig. 4

Puede apreciarse que las velocidades de corrosión detectadas son de 10 a 100 veces menores que con el acero para hormigón armado, y que varía también el orden de clasificación por capacidad protectora de los distintos cementos. Estos resultados indican que es fundamental utilizar siempre materiales y condiciones de ensayo idénticas, y que como se aludía en la introducción es necesario considerar en cada caso el conjunuto cemento/ /hormigón/acero, más durable.

\section{DISCUSION}

Se ha presentado el resumen de tres ensayos realizados con diferentes series de cementos, para poner de manifiesto principalmente que las técnicas electroquímicas utilizadas permiten en cierta medida obtener una información valiosa sobre el diferente comportamiento de los distintos cementos y tipos de acero ensayados, frente a un ataque por corrosión. 
Estos ensayos no son los únicos efectuados por los autores sobre la problemática de la influencia del tipo de cemento en una posible corrosión de las armaduras, ya que trabajan en esta línea desde 1970 habiendo publicado algunos resultados (12) (16) (17) y comentarios teóricos (18) (19) y manteniendo otros aún sin publicar.

A partir de los resultados aquí presentados y a través de la experiencia de más de 10 años mencionada se ha podido deducir que, si el medio no es agresivo, las armaduras de acero se pasivan perfectamente en contacto con todos los cementos ensayados, contengan o no adiciones activas. Unicamente, las armaduras embebidas en cementos con escorias en proporciones superiores al $40-50 \%$, se pasivan más lentamente y, cuando el mortero se conserva húmedo, en ocasiones aparece una ligera corrosión localizada.

Ahora bien, cuando se desencadena un ataque originado por otras causas (la presencia de un $2 \%$ de $\mathrm{Cl}_{2} \mathrm{Ca}$ en el presente caso), entonces los diversos cementos desarrollan frente a los $\mathrm{Cl}^{-}$una capacidad regenerante de la capa pasiva diferente, siendo en general los portland puros los que resultan más protectores, y los menos, los que contienen escorias siderúrgicas.

En síntesis, se puede decir que los cementos con adiciones activas resultan en general "potencialmente" menos protectores para las armaduras, ya que aumentan, tanto la probabilidad de que se produzca una corrosión como su cuantía, si se inicia el ataque. Es importante añadir que se han encontrado algunos cementos con adición activa, que resultan tan protectores como los portland puros, y algunos portland comerciales que no se comportan como puros.

El otro factor considerado en los presentes ensayos es la influencia del estado de rugosidad superficial del acero. Se observa comparando las figuras 3 y 4 (y en otros ensayos efectuados con anterioridad pero aún no publicados) que, mientras una determinada proporción de cloruros pueden no provocar ningún ataque en un acero liso y pulido embebido en un determinado cemento, en cambio la misma proporción puede resultar muy agresiva para un acero corrugado embebido en el mismo mortero. Lo que lleva a reiterar que en el comportamiento de la armadura influye también decisivamente el tipo y acabado superficial del acero, así como su estado de conservación, por lo que la durabilidad de las armaduras debe ser considerada globalmente en función de todas las variables potencialmente influyentes.

\section{B I B L I O G R A F I A}

(1) P. LoNGUET: Silicates Industriels, $7 / 8$ (1976) 321.

(2) P. Delmas: Rev. Matériaux et Constr. 5 (1976) 291.

(3) P. Peguin, M. Rubaud, P. Longuet et A. Zelwer: Cahiers du Centre Scient. et Techn. du Bâtim n. 130 Jun. (1972).

(4) Building Code of the American Concrete Institute. (1978).

(5) F.I.P.: Technical Report on Prestressing Steel n." 6 Dic. (1980).

(6) RILEM-12-CRC Committee: Matér. et Const. 9 (1976) 187.

(7) V. Solana: Hormigón y Acero n.” 119-120 (1976) 10. 
(8) J. A. Gonzalez et C. Andrade: Brit. Corros. Jcurnal 3 (1980) 135.

(9) C. Andrade, G. Jauregui et J. A. Gonzalez: 8." Int. Cong. of Metall. Corros. Mainz Oct. (1981).

(10) C. Andrade et J. A. Gonzalez: Cuaderno de Invest. del IETCC n.”33 Junio (1978).

(11) C. Andrade et J. A. Gonzalez: Werkstoffe und Korros. 29 (1978) 515.

(12) C. Andrade: Tesis Doctoral. Univ. Complutense de Madrił. Julio (1973).

(13) H. KAESCHE: Zement-Kalk-Gips. 12,7 (1959) 289.

(14) H. KAESCHE: Archiv. Eisenhuttenw. 36 (1965) 911.

(15) A. BAUMeL: Zement-Kalk-Gips. 12,7 (1959) 294.

(16) C. Andrade: Trabajo de Licenciatura. Universidad Complutense de Madrid. Junio 1970.

117) C. Andrade: Corrosión y Protección. Marzo (1977) pág. 38.

(18) C. ANdrade: Hormigón y Acero n." 125 (1977) pág. 128.

19) C. Andrade: Materiales de Construcción n.’ 172 (1978) pág. 63. 